\title{
The Generalized Good Cut Equation
}

\author{
T.M. Adamo* \\ Mathematical Institute \\ University of Oxford \\ 24-29 St Giles \\ Oxford, OX1 3LB, UK
}

\author{
E.T. Newman ${ }^{\dagger}$ \\ Department of Physics \& Astronomy \\ University of Pittsburgh \\ 3941 O'Hara Street \\ Pittsburgh, PA 15260, USA
}

October 25, 2018

\begin{abstract}
The properties of null geodesic congruences (NGCs) in Lorentzian manifolds are a topic of considerable importance. More specifically NGCs with the special property of being shear-free or asymptotically shear-free (as either infinity or a horizon is approached) have received a great deal of recent attention for a variety of reasons. Such congruences are most easily studied via solutions to what has been referred to as the 'good cut equation' or the 'generalization good cut equation'. It is the purpose of this note to study these equations and show their relationship to each other. In particular we show how they all have a four complex dimensional manifold (known as $\mathcal{H}$-space, or in a special case as complex Minkowski space) as a solution space.
\end{abstract}

\section{Contents}

1 Introduction 2

2 The Good Cut Equations 3

2.1 Solution Space of the GCEq . . . . . . . . . . . . . . . 4

2.2 The Good Cut Equation and Twistors . . . . . . . . . . . 5

3 Equivalence of Good Cut Equations: From the $\mathrm{G}^{2} \mathrm{CEq}$ to the GCEq 9

3.1 Application: Vacuum Non-Expanding Horizons . . . . . . . . . . . . . 10

4 Conclusion 11

*adamo@maths.ox.ac.uk

$\dagger^{\dagger}$ newman@pitt.edu 


\section{Introduction}

Shear-free (and its generalization to asymptotically shear-free) null geodesic congruences (NGCs) in Lorentzian space-times have played a variety of important roles in space-time geometry. They first appeared in the search for algebraically special solutions of Maxwell's equations in curved space-times [15. This was followed by the discovery of the vacuum twist-free algebraically special metrics of Robinson and Trautman 16 and the extension, via the remarkable Goldberg-Sachs theorem [6, to all algebraically special vacuum metrics. Penrose then showed the close connection of shear-free congruences, via the Kerr theorem, with twistor theory [13. More recently the asymptotically shear-free congruences (as null infinity is approached) have been used to give physical interpretations to the asymptotic fields in asymptotically flat space-times [1. This latter case has been generalized to congruences that become shear-free as a non-expanding horizon in a space-time is approached [2].

All the cases are governed by solutions to different versions of the same type of partial differential equation, referred to as "good cut equations." The good-cut equation (GCEq) or its generalization, the generalized GCEq $\left(\mathrm{G}^{2} \mathrm{CEq}\right)$, are second order PDEs that live on 3 -manifolds. Although it is very likely that the discussion could be generalized to arbitrary 3 -surfaces in a Lorentzian manifold (or more specifically to arbitrary null surfaces), we confine ourselves to the cases where the 3 -surfaces are specifically Penrose's future null infinity, $\mathfrak{I}^{+}$, or a vacuum non-expanding horizon, $\mathfrak{H}$ (c.f., 4, 5]). For the general discussion we will refer to both $\mathfrak{I}$ and/or $\mathfrak{H}$ as $\mathfrak{N}$. In each of these cases $\mathfrak{N}$ has topology $S^{2} \times \mathbb{R}$, and is foliated by null geodesics congruences whose shear and divergence both vanish on $\mathfrak{N}$. Though these manifolds are real 3-surfaces in the real space-time, we must consider their complexification, $\mathfrak{N}_{\mathbb{C}}$ (i.e., their analytic continuation, at least a small way, into the complexification of the space-time). The coordinatization of $\mathfrak{N}$ is given by Bondi-like coordinates: $(\zeta, \bar{\zeta})$, which label the null generators of $\mathfrak{N}$, are the stereographic coordinates on the $S^{2}$ portion of $\mathfrak{N}\left(S^{2}\right.$ need not be a metric sphere); while the coordinate $u$ parametrizes the cross-sections of $\mathfrak{N}$. For $\mathfrak{N}_{\mathbb{C}}$, the $u$ is allowed to take complex values close to the real, while $\bar{\zeta}$ goes over to an independent variable $\widetilde{\zeta}$ close to the complex conjugate of $\zeta$. To avoid the notational nuisance of repeatedly saying that $\widetilde{\zeta} \approx \bar{\zeta}$, we will simply use $\bar{\zeta}$ to mean an independent complex variable taking values at, or close to, the complex conjugate of $\zeta$. The context should make its usage clear. The distinction between the GCEq and the $\mathrm{G}^{2} \mathrm{CEq}$ is that the former lives on a 3 -surface $\mathfrak{N}$ whose $u=$ constant cross-sections are metric spheres, while for the latter equation the metric is arbitrary.

In Section 2, we first discuss the geometric meaning of the GCEq and its connection to (asympotically) shearfree NGCs. This is followed by a review of the known properties of solutions to the GCEq: its four complex-dimensional solution space and its relationship to twistor theory. In Section 3 we show that the $\mathrm{G}^{2} \mathrm{CEq}$ can be transformed directly to the GCEq so that the solution spaces of both equations and properties are equivalent. We demonstrate the utility of this result by applying it to the twistor space associated with horizon shear-free NGCs at a non-expanding horizon. Section 4 concludes with a discussion of possible applications of our findings. 


\section{The Good Cut Equations}

Before describing the GCEq we first discuss a notational choice. As mentioned earlier, the 3 -surface $\mathfrak{N}$ is described by an $S^{2}$ worth of null geodesics with the cross sections given by $u=$ constant. The metric of the two-surface cross-sections are expressed in stereographic coordinates $(\zeta, \bar{\zeta})$ so that the metric takes the conformally flat form:

$$
d s^{2}=\frac{4 d \zeta d \bar{\zeta}}{P^{2}(\zeta, \bar{\zeta})}
$$

with $P(\zeta, \bar{\zeta})$ an arbitrary smooth non-vanishing function on the $(\zeta, \bar{\zeta})$, the extended complex plane (Riemann sphere). In the special case of a metric sphere we take

$$
P=P_{0} \equiv 1+\zeta \bar{\zeta}
$$

while in general we write

$$
P=V(\zeta, \bar{\zeta}) P_{0}
$$

The $\mathrm{G}^{2} \mathrm{CEq}$ contains the general $P$, while the special case using $P_{0}$ yields the GCEq.

For the most general situation, the $\mathrm{G}^{2} \mathrm{CEq}$ can be written as a differential equation for the function $u=G(\zeta, \bar{\zeta})$ :

$$
\bar{\partial}^{2} G \equiv \partial_{\bar{\zeta}}\left(V^{2} P_{0}^{2} \partial_{\bar{\zeta}} G\right)=\sigma(G, \zeta, \bar{\zeta})
$$

or

$$
P_{0}^{2} \partial_{\bar{\zeta}}^{2} G+2\left[P_{0}^{2} V^{-1} \partial_{\bar{\zeta}} V+P_{0} \zeta\right] \partial_{\bar{\zeta}} G=V^{-2} \sigma(G, \zeta, \bar{\zeta})
$$

When $V=1$ we have the GCEq:

$$
\overline{\mathrm{\delta}}_{0}^{2} G \equiv \partial_{\bar{\zeta}}\left(P_{0}^{2} \partial_{\bar{\zeta}} G\right)=\sigma(G, \zeta, \bar{\zeta}) .
$$

Further, when the arbitrary spin-weight 2 function $\sigma(G, \zeta, \bar{\zeta})$ vanishes, we have the homogeneous GCEq:

$$
\partial_{\bar{\zeta}}\left(P_{0}^{2} \partial_{\bar{\zeta}} G\right)=0
$$

In the following section it will be shown that the $\mathrm{G}^{2} \mathrm{CEq}$ can, by a (non-obvious) coordinate transformation, be transformed into Eq.(5). We can simply describe the solutions (and its properties) of Eq.(5): it then follows that they also are true for Eq.(4). Chief among these properties is the fact the solution space to (5) is a complex 4-manifold with a natural Einstein metric; hence, taking any curve in this solution space produces a 1-parameter family of good cut functions which in turn describe a foliation of 3 -surface $\mathfrak{N}$.

Remark 2.1 Solutions to the $G C E q$ or $G^{2} C E q, u=G(\zeta, \bar{\zeta})$, known as "good cut functions," describe cross-sections of $\mathfrak{N}$ that are referred to as "good cuts". From the tangent tangents to these good cuts, $L=\bar{\delta} G$, one can construct null directions, (out of $\mathfrak{N}$ ), into the space-time iteself that determine a NGC whose shear vanishes at $\mathfrak{N}$. (See the following section.) When $\mathfrak{N}=\mathfrak{I}^{+}$, these are asymptotically shear-free NGCs; when $\mathfrak{N}$ is a non-expanding horizon, these are vacuum-horizon-shear-free NGCs. 


\subsection{Solution Space of the GCEq}

In this and the following subsection we will be concerned only with the GCEq (5) and its solutions.

The key fact about the solution space to the GCEq is that it forms a complex 4-manifold, known as $\mathcal{H}$-space, for sufficiently regular $\sigma(G, \zeta, \bar{\zeta})$ (which is assumed here). This manifold of solutions can be shown to possess a vacuum Einstein metric with anti-self-dual Weyl tensor. Although the rigorous proof of the existence and properties of $\mathcal{H}$-space requires the use of Kodaira deformation theory and Penrose's non-linear graviton construction [7, we can give a simple intuitive argument here. The solutions will be written as

$$
u=G\left(z^{a}, \zeta, \bar{\zeta}\right),
$$

with $z^{a}$ (appearing as four constants of integration) the $\mathcal{H}$-space coordinates.

Since, from the properties of the (sphere) $\partial_{0}$-operator, one immediately has that the general regular solution of the homogeneous equation (6) is given by the four parameter function

$$
\begin{aligned}
G_{0}\left(z^{a}, \zeta, \bar{\zeta}\right) & =z^{a} l_{a}(\zeta, \bar{\zeta})=\frac{\sqrt{2}}{2} z^{0}+\frac{1}{2} z^{i} Y_{1 i}^{0}(\zeta, \bar{\zeta}) \\
l_{a}(\zeta, \bar{\zeta}) & =\frac{\sqrt{2}}{2 P_{0}}(1+\zeta \bar{\zeta}, \zeta+\bar{\zeta}, i \bar{\zeta}-i \zeta,-1+\zeta \bar{\zeta})
\end{aligned}
$$

The inhomogeneous equation can then be rewritten as the integral equation

$$
G=G_{0}\left(z^{a}, \zeta, \bar{\zeta}\right)+\int \sigma^{0}(G, \eta, \bar{\eta}) K_{0,-2}^{+}(\eta, \bar{\eta}: \zeta, \bar{\zeta}) d S_{\eta},
$$

with

$$
\begin{aligned}
K_{0,-2}^{+}(\zeta, \bar{\zeta}, \eta, \bar{\eta}) & \equiv-\frac{1}{4 \pi} \frac{(1+\bar{\zeta} \eta)^{2}(\eta-\zeta)}{(1+\zeta \bar{\zeta})(1+\eta \bar{\eta})(\bar{\eta}-\bar{\zeta})} \\
d S_{\eta} & =4 i \frac{d \eta \wedge d \bar{\eta}}{(1+\eta \bar{\eta})^{2}}
\end{aligned}
$$

where $K_{0,-2}^{+}(\zeta, \tilde{\zeta}, \eta, \tilde{\eta})$ is the Green's function for the $\partial_{0}^{2}$-operator 9 . By iterating this equation, with $G_{0}\left(z^{a}, \zeta, \tilde{\zeta}\right)=z^{a} l_{a}(\zeta, \bar{\zeta})$ being the zeroth iterate,

$$
G_{n}(\zeta, \bar{\zeta})=z^{a} l_{a}(\zeta, \bar{\zeta})+\int_{S^{2}} K_{0,-2}^{+}(\zeta, \bar{\zeta}, \eta, \bar{\eta}) \sigma\left(G_{n-1}, \eta, \bar{\eta}\right) d S_{\eta},
$$

one easily sees how the four $z^{a}$ enter the solution: the four constants originate from the solution to the homogeneous equation.

We thus have the result that the solutions to the GCEq.

$$
u=G\left(z^{a}, \zeta, \bar{\zeta}\right),
$$

defines a four-parameter family of cuts of $\mathfrak{N}$, each cut labeled by the $\mathcal{H}$-space points, $z^{a}$. By choosing an arbitrary analytic curve in the $\mathcal{H}$-space, $z^{a}=\xi^{a}(\tau)$, with $\tau$ an arbitrary complex parameter, we have a one-parameter family of cuts of $\mathfrak{N}$ 


$$
u=Z(\tau, \zeta, \bar{\zeta})=G\left(\xi^{a}(\tau), \zeta, \bar{\zeta}\right) .
$$

Each choice of the curve $z^{a}=\xi^{a}(\tau)$ yields an asympotically shear-free NGC by the following construction:

Considering $\mathfrak{N}$ as embedded in a Lorentzian space-time, at each point of $\mathfrak{N}$ we can construct its past (or future) light-cone. The sphere of null directions can be coordinitized by complex stereographic coordinates, $(L, \bar{L})$. A field of null directions pointing backward (or forward) from $\mathfrak{N}$ can be written as

$$
L=L(\mathfrak{N})=L(u, \zeta, \bar{\zeta}) .
$$

It is known that the field of null directions that is given parametrically on $\mathfrak{N}$ by

$$
\begin{aligned}
L(u, \zeta, \bar{\zeta}) & =\partial_{0} Z(\tau, \zeta, \bar{\zeta}), \\
u & =Z(\tau, \zeta, \bar{\zeta}),
\end{aligned}
$$

describes the null direction field of an asymptotically shearfree NGC 3, 12, 11, 1,. When we are dealing with the homogeneous equation on $\mathfrak{I}$ in Minkowski space, the NGC turns out to be shear-free everywhere. 12

We point out that the solution space comes naturally with the $(\mathcal{H}$-space) complex metric

$$
\begin{aligned}
d s_{(\mathcal{H})}^{2} & =g_{(\mathcal{H}) a b} d z^{a} d z^{b} \equiv\left(\frac{1}{8 \pi} \int_{S^{2}} \frac{d S}{(d G)^{2}}\right)^{-1} \\
d G & =\nabla_{a} G d z^{a} \\
d S & =4 i \frac{d \zeta \wedge d \bar{\zeta}}{(1+\zeta \bar{\zeta})^{2}}
\end{aligned}
$$

that is Ricci flat and has anti-self-dual conformal (Weyl) curvature. For the special case of solutions to the homogeneous GCEq, this metric reduces to the complex Minkowski metric.

\subsection{The Good Cut Equation and Twistors}

The study of the GCEq is intimately related to Penrose's twistor theory. Although we will not provide an extensive review of twistor theory here, we will include the briefest of overviews to set the stage for the following discussion. The interested reader need only consult 8, 14, for a more in-depth introduction and discussion. For our purposes, twistor space is the complex projective 3 -space $\mathbb{P T} \simeq \mathbb{C P}^{3}$, charted with homogeneous coordinates $Z^{\alpha}=\left(\omega^{A}, \pi_{A^{\prime}}\right)$, where $\omega$ and $\pi$ are un-primed and primed Weyl spinors respectively. A (projective) twistor is any point $Z^{\alpha} \in \mathbb{P} \mathbb{T}$. Twistor space is related to points $x$ in complex Minkowski space-time by the incidence relation:

$$
\omega^{A}=i x^{A A^{\prime}} \pi_{A^{\prime}},
$$


where $x^{A A^{\prime}}$ is the usual spinor representation (where a vector index is replaced by a pair of primed and un-primed spinor indicies),

$$
x^{A A^{\prime}}=\frac{1}{\sqrt{2}}\left(\begin{array}{cc}
t+x & y-i z \\
y+i z & t-x
\end{array}\right) .
$$

Eq. (16) can be used to determine that a point in $\mathbb{P T}$ corresponds to a null geodesic in complex Minkowski space, while a point $x$ in complex Minkowski space corresponds to a line $L_{x} \simeq \mathbb{C P}^{1} \subset \mathbb{P T}$. The geometry of real Minkowski space-time is recovered on null twistor space, defined as

$$
\mathbb{P N}=\left\{Z^{\alpha} \in \mathbb{P} \mathbb{T}: \omega^{A} \bar{\pi}_{A}+\pi^{A^{\prime}} \bar{\omega}_{A^{\prime}}=0\right\} .
$$

In other words, $Z^{\alpha}$ corresponds to a real null geodesic in Minkowski space-time if and only if $Z^{\alpha} \in \mathbb{P N}$; and $L_{x}$ corresponds to a real point in Minkowski space-time if and only if $L_{x} \subset \mathbb{P N}$.

We can also chart $\mathbb{P} \mathbb{T}$ with non-homogeneous coordinates, which are most useful when studying the GCEq. Assuming that $\pi_{A^{\prime}} \neq 0$ (which corresponds to excluding the point at infinity by (16) ) and working on a patch where $\pi_{0^{\prime}} \neq 0$, we can write $[10$ :

$$
\left(\omega^{A}, \pi_{A^{\prime}}\right)=\left(i r \mu^{0},-i r \mu^{1}, r,-r \zeta\right)
$$

where $r \in \mathbb{C}$ is a common scaling factor and $\zeta$ can be shown to be equivalent to the complex stereographic angle on $S^{2}$ introduced earlier. This means that $\left(\zeta, \mu^{0}, \mu^{1}\right)$ can be interpreted as coordinates on $\mathbb{P} \mathbb{T} \cong \mathbb{C P}^{3}$ via

$$
\begin{aligned}
\zeta & =-\frac{\pi_{1^{\prime}}}{\pi_{0^{\prime}}}, \\
\mu^{0} & =-i \frac{\omega^{0}}{\pi_{0^{\prime}}}, \\
\mu^{1} & =i \frac{\omega^{1}}{\pi_{0^{\prime}}} .
\end{aligned}
$$

A curved twistor space $\mathbb{P} \mathcal{T}$ can also be constructed for any complex space-time that is Ricci flat with anti-self-dual conformal curvature by the non-linear graviton construction (c.f., [8]). Such curved twistor spaces have a similar correspondence with null geodesics and points in the complex space-time, although the curves $L_{x}$ will no longer be lines, as in (16).

We now discuss the relationship between the above discussion of twistor space and the GCEq.

Starting with the homogeneous GCEq, twistor space can be constructed in the following manner. Treating the variable $\zeta=\zeta_{0}$ for the moment as a fixed constant, the homogeneous GCEq (6) becomes a second-order ODE for $u=G(\bar{\zeta})$. Its solution is determined by two initial conditions: the value of $G$ and its first derivative at $\bar{\zeta}$ equal to the complex conjugate of $\zeta$ (denoted by $\left.\bar{\zeta}_{0}\right)$, i.e., at $u_{0}=G\left(\bar{\zeta}_{0}\right)$ and $L_{0}=\left(1+\bar{\zeta}_{0} \zeta_{0}\right) \partial_{\bar{\zeta}_{0}} G\left(\bar{\zeta}_{0}\right)$. The curves sodetermined are defined as a projective twistors, with $\mathbb{P T}$ being the collection of all such curves. We then adopt $\left(\zeta_{0}, u_{0}, L_{0}\right)$ as local coordinates on $\mathbb{P T}$, with the relationship to the standard twistor coordinates given earlier by

$$
\left(\zeta_{0}, \mu_{0}, \mu_{1}\right)=\left(\zeta_{0}, u_{0}-\bar{\zeta}_{0} L_{0}, \zeta_{0} u_{0}+L_{0}\right) .
$$


These relations come directly from the integration of the ODE:

$$
\begin{aligned}
\partial_{\bar{\zeta}}\left(1+\zeta_{0} \bar{\zeta}\right)^{2} \partial_{\bar{\zeta}} G & =0 \\
\partial_{\bar{\zeta}} G & =\left(1+\zeta_{0} \bar{\zeta}\right)^{-2} \alpha_{0}, \\
\bar{L} & =\bar{\delta} G \equiv\left(1+\zeta_{0} \bar{\zeta}\right) \partial_{\bar{\zeta}} G=\left(1+\zeta_{0} \bar{\zeta}\right)^{-1} \alpha_{0} \\
& \Longrightarrow u=G=\alpha_{1}-\zeta_{0}^{-1}\left(1+\zeta_{0} \bar{\zeta}\right)^{-1} \alpha_{0}
\end{aligned}
$$

The pair of integration constants, $\left(\alpha_{0}, \alpha_{1}\right)$, are determined directly in terms of the initial conditions $\left(u_{0}, L_{0}\right)$. Defining $\left(\mu_{0}, \mu_{1}\right)$ by

$$
\alpha_{0}=\mu_{1}-\zeta \mu_{0}, \quad \alpha_{1}=\mu_{1} \zeta^{-1},
$$

we have

$$
\begin{aligned}
u & =\left(\mu_{1} \bar{\zeta}+\mu_{0}\right)\left(1+\zeta_{0} \bar{\zeta}\right)^{-1} \\
\bar{L} & =\left(\mu_{1}-\zeta_{0} \mu_{0}\right)\left(1+\zeta_{0} \bar{\zeta}\right)^{-1} \\
u_{0} & =\left(\mu_{1} \bar{\zeta}_{0}+\mu_{0}\right)\left(1+\zeta_{0} \bar{\zeta}_{0}\right)^{-1} \\
\bar{L}_{0} & =\left(\mu_{1}-\zeta_{0} \mu_{0}\right)\left(1+\zeta_{0} \bar{\zeta}_{0}\right)^{-1}
\end{aligned}
$$

Suppose two different twistors were chosen with the local coordinates $\left(\zeta_{0}, u_{0}, L_{0}\right)$ and $\left(\zeta_{1}, u_{1}, L_{1}\right)$, and their respective solution curves $G_{1,2}$ equated with an arbitrary four parameter regular solution of the form (8)

$$
G_{0}\left(z^{a}, \zeta, \bar{\zeta}\right)=z^{a} l_{a}(\zeta, \bar{\zeta})=\frac{\sqrt{2}}{2} z^{0}+\frac{1}{2} z^{i} Y_{1 i}^{0}(\zeta, \bar{\zeta}),
$$

at $\zeta=\zeta_{0}$ and $\zeta=\zeta_{1}$. This yields four linear algebraic equations to determine the four coordinates $z^{a}$ in terms of the four $\left(u_{0}, L_{0}, u_{1}, L_{1}\right)$. This construction is totally equivalent to the use of the twistor incidence relationship (16) to determine the space-time points $x^{A A^{\prime}}$ from a pair of projective twistors, since the two twistors $\left(\zeta_{0}, u_{0}, L_{0}\right)$ and $\left(\zeta_{1}, u_{1}, L_{1}\right)$ uniquely determine a line $L_{x} \subset \mathbb{P} \mathbb{T}$. This is a linear relationship, in that the choice of any pair of twistors determines both the space-time point $x^{A A^{\prime}}$ and a line in projective twistor space, where any pair of points on the line determine the same point $x^{A A^{\prime}}$.

The attempt to apply this construction to the inhomogeneous equation, Eq.(5) fails; the relationship is no longer linear. One obtains instead a differential equation describing a (non-linear) curve in a curved twistor space such that any point and its tangent vector on the curve determines a point in $\mathcal{H}$-space. The argument we give for this is very heuristic in the sense we are assuming (without proof) that several implicit algebraic equations can be inverted. When the situation is sufficiently close to that of the homogeneous equation (i.e., for suitably small $\sigma(u, \zeta, \bar{\zeta})$ ), this should not be a problem.

By rewriting Eq.(5) as

$$
P_{0}^{2} \partial_{\bar{\zeta}} \partial_{\bar{\zeta}} G+2 P_{0} \zeta \partial_{\bar{\zeta}} G=\sigma(G, \zeta, \bar{\zeta})
$$

or in the compressed form

$$
\begin{aligned}
\partial_{\bar{\zeta}}^{2} G & =S\left(G, \partial_{\bar{\zeta}} G, \zeta, \bar{\zeta}\right) \\
S & \equiv P_{0}^{-2} \sigma(G, \zeta, \bar{\zeta})-2 P_{0}^{-1} \zeta \partial_{\bar{\zeta}} G
\end{aligned}
$$


we see that there are two different types of solutions:

The first comes from the condition that $\zeta=\zeta_{0}$ is taken as a constant and Eq.(17) can be treated as a second order ODE for $G$ as a function of $\bar{\zeta}$ whose solution depends on two constants of integration, $(\alpha, \beta)$. They can be taken as the initial value and first derivative of $G$ at some arbitrary point $\bar{\zeta}=\widetilde{\zeta}_{0}$. On a fiducial (or special) curve we take $\widetilde{\zeta}_{0}$ to be the complex conjugate of $\zeta_{0}: \widetilde{\zeta}_{0}=\bar{\zeta}_{0}$. The solution will then be written as

$$
u=G^{(1)}\left(\alpha\left(\bar{\zeta}_{0}\right), \beta\left(\bar{\zeta}_{0}\right), \zeta_{0}, \bar{\zeta}\right) .
$$

This curve in $(u, \bar{\zeta})$ space, labeled by $\left(\alpha, \beta, \zeta_{0}\right)$, is identified as a twistor with local coordinates $\left(\alpha\left(\bar{\zeta}_{0}\right), \beta\left(\bar{\zeta}_{0}\right), \zeta_{0}\right)$. (It should be noted that if the initial value point, $\bar{\zeta}_{0}$, on the twistor curve is changed, the new values of $\alpha$ and $\beta$ are easily found.) By freeing up $\zeta_{0}$, i.e., allowing $\zeta_{0} \rightarrow \zeta$, to vary and letting $\alpha, \beta$ be functions of both $\zeta$ and the fixed initial value point $\bar{\zeta}_{0}$, we obtain a one-parameter family of twistors (or a twistor-space curve),

$$
u=G^{(1)}\left(\alpha\left(\zeta, \bar{\zeta}_{0}\right), \beta\left(\zeta, \bar{\zeta}_{0}\right), \zeta, \bar{\zeta}\right) .
$$

The second type of solutions are the regular ones, eq.(13), that depend on four constants, the $\mathcal{H}$-space coordinates, $z^{a}$ :

$$
u=G^{(2)}\left(z^{a}, \zeta, \bar{\zeta}\right) .
$$

Holding the $z^{a}$ fixed and varying $\zeta$, we obtain a one-parameter family of twistors (i.e. a curve in $\mathbb{P} \mathcal{T}$ ).

The question is: how can the two functions $\alpha\left(\zeta, \bar{\zeta}_{0}\right), \beta\left(\zeta, \bar{\zeta}_{0}\right)$ be chosen so that two sets of solutions coincide?

This is accomplished by first equating the two types of solution and their first $\bar{\zeta}$ derivatives at $\bar{\zeta}=\bar{\zeta}_{0}$ :

$$
\begin{aligned}
G^{(1)}\left(\alpha\left(\zeta, \bar{\zeta}_{0}\right), \beta\left(\zeta, \bar{\zeta}_{0}\right), \zeta, \bar{\zeta}_{0}\right) & =G^{(2)}\left(z^{a}, \zeta, \bar{\zeta}_{0}\right), \\
\partial_{\bar{\zeta}} G^{(1)}\left(\alpha\left(\zeta, \bar{\zeta}_{0}\right), \beta\left(\zeta, \bar{\zeta}_{0}\right), \zeta, \bar{\zeta}_{0}\right) & =\partial_{\bar{\zeta}} G^{(2)}\left(z^{a}, \zeta, \bar{\zeta}_{0}\right) .
\end{aligned}
$$

We thus have a pair of implicit equations whose algebraic solution for $\alpha$ and $\beta$ has the form:

$$
\begin{aligned}
& \alpha\left(\zeta, \bar{\zeta}_{0}\right)=A\left(z^{a}, \zeta, \bar{\zeta}_{0}\right) \\
& \beta\left(\zeta, \bar{\zeta}_{0}\right)=B\left(z^{a}, \zeta, \bar{\zeta}_{0}\right) .
\end{aligned}
$$

It is easy to derive, by the following argument, a (parametric) pair of second order ODEs for $\alpha$ and $\beta$ where (23) 24) are the solutions and $z^{a}$ are the constants of integration. First take the $\zeta$ derivative of (23) and (24):

$$
\begin{aligned}
& \alpha^{\prime}\left(\zeta, \bar{\zeta}_{0}\right)=\partial_{\zeta} A\left(z^{a}, \zeta, \bar{\zeta}_{0}\right), \\
& \beta^{\prime}\left(\zeta, \bar{\zeta}_{0}\right)=\partial_{\zeta} B\left(z^{a}, \zeta, \bar{\zeta}_{0}\right) .
\end{aligned}
$$

The two pairs, Eqs.(23]|24) and (25]|26), determine implicitly

$$
z^{a}=\mathcal{Z}^{a}\left(\alpha, \beta, \alpha^{\prime}, \beta^{\prime}, \zeta, \bar{\zeta}_{0}\right)
$$


Finally taking the $\zeta$ derivative of (25) and (26):

$$
\begin{aligned}
& \alpha^{\prime \prime}\left(\zeta, \bar{\zeta}_{0}\right)=\partial_{\zeta} \partial_{\zeta} A\left(z^{a}, \zeta, \bar{\zeta}_{0}\right), \\
& \beta^{\prime \prime}\left(\zeta, \bar{\zeta}_{0}\right)=\partial_{\zeta} \partial_{\zeta} B\left(z^{a}, \zeta, \bar{\zeta}_{0}\right),
\end{aligned}
$$

and eliminating $z^{a}$, via (27), leaves the pair

$$
\begin{aligned}
& \alpha^{\prime \prime}\left(\zeta, \bar{\zeta}_{0}\right)=\mathcal{A}\left(\alpha, \beta, \alpha^{\prime}, \beta^{\prime}, \zeta, \bar{\zeta}_{0}\right), \\
& \beta^{\prime \prime}\left(\zeta, \bar{\zeta}_{0}\right)=\mathcal{B}\left(\alpha, \beta, \alpha^{\prime}, \beta^{\prime}, \zeta, \bar{\zeta}_{0}\right) .
\end{aligned}
$$

The solutions determine, for each set of constants, $z^{a}$, a curve in the asymptotic twistor space.

Though it might be (and probably should be) possible to construct the $\mathcal{A}$ and $\mathcal{B}$ directly from the GCEq. (i.e., from $\sigma(u, \zeta, \bar{\zeta})$ ), we do not at the present know how to do this. Nevertheless it is nice to see that such curves do exist and determine points in the $\mathcal{H}$-space. Hence, we have confirmed precisely what is known from twistor theory: a point in $\mathcal{H}$-space (a vacuum, anti-self-dual complex space-time) corresponds to a curve in a curved twistor space. Indeed, since $\mathcal{H}$-space reduces to complex Minkowski space in the case of the homogeneous GCEq, we saw (as expected) that points in this trivial $\mathcal{H}$-space corresponded to lines in $\mathbb{P} T$.

\section{Equivalence of Good Cut Equations: From the $\mathrm{G}^{2} \mathrm{CEq}$ to the GCEq}

In this section we show how, by a coordinate transformation of the (independent) complex stereographic coordinates $(\zeta, \bar{\zeta})$, the generalized GCEq $\left(\mathrm{G}^{2} \mathrm{CEq}\right)$ can be transformed into the GCEq. It must be remembered from our notation that $\bar{\zeta}^{*}$ (or $\bar{\zeta}$ ) is close to, but is not necessarily, the complex conjugate of $\zeta^{*}$ (or $\zeta$ ).

We first rewrite the GCEq with stereographic coordinates $\left(\zeta^{*}, \bar{\zeta}^{*}\right)$ as

$$
\begin{aligned}
\bar{ळ}_{0 *}^{2} G & =\partial_{\bar{\zeta}^{*}}\left(P_{0}^{* 2} \partial_{\bar{\zeta}^{*}} G\right)=\sigma^{*}\left(G, \zeta^{*}, \bar{\zeta}^{*}\right), \\
P_{0}^{*} & =1+\zeta^{*} \bar{\zeta}^{*},
\end{aligned}
$$

and the $\mathrm{G}^{2} \mathrm{CEq}$ as

$$
\bar{\delta}^{2} G=\partial_{\bar{\zeta}}\left(V^{2} P_{0}^{2} \partial_{\bar{\zeta}} G\right)=\sigma(G, \zeta, \bar{\zeta}) .
$$

We now apply the coordinate transformation

$$
\begin{aligned}
\bar{\zeta}^{*} & =\frac{\bar{\zeta}+W}{1-W \zeta} \equiv N(\zeta, \bar{\zeta}), \\
\zeta^{*} & =\zeta
\end{aligned}
$$

with $W$ (a spin-weight 1 function) defined from

$$
\begin{aligned}
V^{-2} & =1+\overline{\mathrm{\partial}}_{0} W=1+P_{0} \partial_{\bar{\zeta}} W-W \zeta \\
P_{0} & =1+\zeta \bar{\zeta}
\end{aligned}
$$


to Eq.(34). Substituting the derived relations,

$$
\begin{aligned}
P_{0}^{*} & =1+\zeta \bar{\zeta}^{*}=\frac{1+\zeta \bar{\zeta}}{1-W \zeta}=\frac{P_{0}}{1-W \zeta}, \\
\partial_{\bar{\zeta}} G & =\partial_{\bar{\zeta}^{*}} G \cdot \partial_{\bar{\zeta}} N \\
\partial_{\bar{\zeta}}^{2} G & =\partial_{\bar{\zeta}^{*}}^{2} G \cdot\left(\partial_{\bar{\zeta}} N\right)^{2}+\partial_{\bar{\zeta}^{*}} G \cdot \partial_{\bar{\zeta}}^{2} N, \\
\partial_{\bar{\zeta}} N & =\frac{V^{-2}-W \zeta}{(1-W \zeta)^{2}}, \\
\partial_{\bar{\zeta}}^{2} N & =\frac{2 \zeta\left[V^{-2}-1\right] \partial_{\bar{\zeta}} W}{(1-W \zeta)^{3}}+\frac{\zeta \partial_{\bar{\zeta}} W}{(1-W \zeta)^{2}}-\frac{2 V^{-3} \partial_{\bar{\zeta}} V}{(1-W \zeta)^{2}}
\end{aligned}
$$

into Eq.(34), we have, after a bit of algebra,

$$
\bar{\delta}_{0 *}^{2} G=\partial_{\bar{\zeta}^{*}}\left(P_{0}^{* 2} \partial_{\bar{\zeta}^{*}} G\right)=F\left(\zeta^{*}, \bar{\zeta}^{*}\right) \sigma\left(G, \zeta\left(\zeta^{*}, \bar{\zeta}^{*}\right), \bar{\zeta}^{*}\left(\left(\zeta^{*}, \bar{\zeta}^{*}\right)\right) \equiv \sigma^{*}\left(G, \zeta^{*}, \bar{\zeta}^{*}\right),\right.
$$

namely Eq. (32), the GCEq.

Hence, we see that the $\mathrm{G}^{2} \mathrm{CEq}$ is really equivalent to the GCEq via the coordinate transformation (35). This means that the study of the $\mathrm{G}^{2} \mathrm{CEq}$ on a general 3-surface $\mathfrak{N}$ can be reduced to the study of the properties of the GCEq on a 3-surface whose cross-sections are metric spheres. Although the form of the coordinate transformation is far from obvious, this equivalence is not totally un-expected, since the good cut equation is known to be conformally invariant.

\subsection{Application: Vacuum Non-Expanding Horizons}

In [2, it was shown that the condition for a neighborhood of a vacuum non-expanding horizon $\sqrt[51]{1}$ to be foliated by a null geodesic congruence whose shear vanishes at the horizon takes the form of a time-independent $\mathrm{G}^{2} \mathrm{CEq}$ :

$$
\check{\partial}^{2} G=\sigma(\zeta, \bar{\zeta})
$$

where the right-hand side does not depend on $u$. It isn't hard to prove that the solution space to this equation is a complex 4-manifold, but it is unclear if this solution space posesses the ordinary $\mathcal{H}$-space metric. Equivalently, we want to know: is there a twistor space associated with the generalized good cut equation on the horizon $\mathfrak{H}$ ?

Answering this question is now almost trivial in light of our previous discussion. Since we continue to work with the complexified surface, a complex supertranslation

$$
u \rightarrow u+f(\zeta, \bar{\zeta})
$$

shifts the function $\sigma$ by Sachs' Theorem:

$$
\sigma(\zeta, \bar{\zeta}) \rightarrow \sigma(\zeta, \bar{\zeta})+\partial^{2} f(\zeta, \bar{\zeta})
$$

\footnotetext{
${ }^{1} \mathrm{~A}$ vacuum non-expanding horizon is a null 3-submanifold in a Lorentzian space-time which has vanishing divergence and shear, is topologically $S^{2} \times \mathbb{R}$, and is fibered over $S^{2}$ by null curves with the vacuum Einstein equations holding in a neighborhood of the horizon.
} 
Hence, we can choose $f$ to set $\sigma=0$ on the entire horizon. This leaves us with the homogeneous $\mathrm{G}^{2} \mathrm{CEq}$ :

$$
\check{\partial}^{2} G=0 .
$$

But by the coordinate transformation (35), we know that this is equivalent to the homogeneous GCEq, which we saw had complex Minkowski space as its solution space. From Section 2.2 , we know that complex Minkowski space corresponds to (flat) twistor space $\mathbb{P} \mathbb{T}$, so it follows that there is a flat twistor space associated with horizon-shear-free NGCs intersecting any vacuum non-expanding horizon.

\section{Conclusion}

In this note we have studied an old topic: the good cut equation and its solution space. In Section 2, we saw how both flat and curved twistor space twistor could be used to explicitly understand the relationship between points in $\mathcal{H}$-space and solutions to the good cut equation on a 3 -surface $\mathfrak{N}$. In the setting where $\mathfrak{N}$ has non-metric sphere cross-sections (such as nonexpanding horizons embedded in space-time), generalized good cut equations arise, which differ from the ordinary GCEq in the definition of the D-operator on the $S^{2}$ portion of the topology. Section 3 demonstrated that these generalized good cut equations are related to the GCEq by a simple coordinate transformation on the complex stereographic coordinates $(\zeta, \bar{\zeta})$. This means that the study of the $\mathrm{G}^{2} \mathrm{CEq}$ on $\mathfrak{N}$ with arbitrary conformal factor on the sphere topology reduces to the study of the normal GCEq on metric 2-spheres. As an example, we saw that this observation immediately implies that the solution space associated to the $\mathrm{G}^{2} \mathrm{CEq}$ on a vacuum non-expanding horizon is complex Minkowski space, and that the corresponding twistor space is the flat $\mathbb{P} T$.

In particular, this indicates that recently developed physical identification theories based on the study of solutions to the GCEq on $\mathfrak{I}^{+}$could be adapted to non-conformal local 3surfaces embedded in space-time (c.f., [1]). In the future, we hope to apply these results to prior studies of non-expanding horizons (e.g., [2]) in the hope of developing a local physical identification theory which could identify physical quantities such as mass, linear momentum, and angular momentum flux at null 3-surfaces in space-time.

\section{References}

[1] Adamo, T. M., Kozameh, C., And Newman, E. T. Null geodesic congruences, asymptotically flat space-times and their physical interpretation. Living Reviews in Relativity 12, 6 (2009).

[2] Adamo, T. M., and Newman, E. T. Vacuum non-expanding horizons and shear-free null geodesic congruences. Classical and Quantum Gravity 26 (2009), 235012.

[3] Aronson, B., And Newman, E. T. Coordinate systems associated with asymptotically shear-free null congruences. Journal of Mathematical Physics 13 (1972), 1847-1851.

[4] Ashtekar, A., Beetle, C., Dreyer, O., Fairhurst, S., Krishnan, B., Lewandowski, J., AND Wisniewski, J. Generic isolated horizons and their applications. Physical Review Letters 85 (2000), 3664-3567. 
[5] Ashtekar, A., Beetle, C., And Lewandowski, J. Geometry of generic isolated horizons. Classical and Quantum Gravity 19 (2002), 1195-1225.

[6] Goldberg, J., And Sachs, R. A theorem on Petrov types. Acta Physics, Pol. 22 (1962), 13-23.

[7] Hansen, R., Newman, E. T., Penrose, R., and Tod, K. P. The metric and curvature properties of $\mathcal{H}$-space. Proceedings of the Royal Society of London, Ser. A. 363 (1978), 445-468.

[8] Hugget, S. A., And Tod, K. P. An Introduction to Twistor Theory. Cambridge University Press, 1985.

[9] Ivancovich, J., Kozameh, C., and Newman, E. T. Green's functions of the $\partial$ operators. Journal of Mathematical Physics 30 (1989), 45-52.

[10] Ko, M., Newman, E. T., and Penrose, R. The Kähler structure of asymptotic twistor space. Journal of Mathematical Physics 18 (1977), 58-64.

[11] Kozameh, C., Newman, E., Santiago-Santiago, J., and Silva-Ortigoza, G. The universal cut function and type II metrics. Classical and Quantum Gravity 24 (2007), 1955-1979.

[12] Newman, E. T. Heaven and its properties. General Relativity and Gravitation 7 (1976), $107-111$.

[13] Penrose, R. Twistor algebra. Journal of Mathematical Physics 8 (1967), 345-366.

[14] Penrose, R., And Rindler, W. Spinors and Space-time, vol. 2. Cambridge University Press, 1986.

[15] Robinson, I. Null electromagnetic fields. Journal of Mathematical Physics 2 (1961), $290-291$.

[16] Robinson, I., And Trautman, A. Some spherical gravitational waves in general relativity. Proceedings of the Royal Society of London, Ser. A. 265 (1962), 463-473. 